\title{
Elaboração e validação de escala diagramática para avaliação da severidade do carvão da folha do caupi
}

\author{
Sami J. Michereff ${ }^{1,2}$, Domingos E.G.T. Andrade ${ }^{1}$, Marissônia A. Noronha ${ }^{1}$
}

${ }^{1}$ Departamento de Agronomia - Área de Fitossanidade, Universidade Federal Rural de Pernambuco, CEP 52171-900, Recife-PE, Brasil; ${ }^{2}$ Bolsista de Produtividade em Pesquisa do CNPq.

Autor para correspondência: Sami J. Michereff. <sami@ufrpe.br>

Data da chegada: 25/06/04. Aceito para publicação em: 16/06/05.

\begin{abstract}
Michereff, S.J.; Andrade, D.E.G.T.; Noronha, M.A. Elaboration and validation of diagrammatic key to evaluate leaf smut severity in cowpea. Summa Phytopathologica, v.32, p. 51-56, 2006.

The leaf smut, caused by the fungus Entyloma vignae, is a common disease of cowpea in the Brazilian Northeast. Considering the inexistence of standard methods for assessment of this disease, a diagrammatic key was developed with 1.5, 3.5, 7.0, 14.5, 27.0 and $45.0 \%$ of diseased leaf areas and tested for accuracy, precision and reproducibility of the estimatives of leaf smut severity with and without using the key. The diagrammatic key was validated by 10 raters, with and without the key, using 48 cowpea leaflets with different levels of severity previously measured by the software AutoCAD ${ }^{\circledR}$ for Windows. Two evaluations were performed with the diagrammatic key at 7-day

interval where different sequences of the same leaves were visually estimated by the same raters. The accuracy and precision of each rater were determined by simple linear regression between actual, evaluated electronically, and estimated severity. Without the diagrammatic key most of raters overestimated disease severity. With the key raters obtained better levels of accuracy and precision, with absolute errors concentrated around $10 \%$. Raters showed good repeatability (90\%) and reproducibility (84\%) of estimative by using the key. The proposed diagrammatic key was suitable for the evaluation of severity of leaf smut on cowpea.
\end{abstract}

Additional keywords: Vigna unguiculata, Entyloma vignae, leaf disease, pathometry.

\section{RESUMO}

Michereff, S.J.; Andrade, D.E.G.T.; Noronha, M.A. Elaboração e validação de escala diagramática para avaliação da severidade do carvão da folha do caupi. Summa Phytopathologica, v.32, p. 51-56, 2006.

O carvão da folha, causado pelo fungo Entyloma vignae, é uma doença comum do caupi no Nordeste brasileiro. Considerando a inexistência de métodos padronizados para quantificação dessa doença, foi elaborada uma escala diagramática com os níveis 1,5 ; 3,$5 ; 7,0 ; 14,5 ; 27,0$ e 45,0\% de área foliar lesionada, testando-se a acurácia, a precisão e a reprodutibilidade das estimativas de severidade do carvão da folha com e sem a utilização desta. Na validação da escala diagramática, 48 folíolos de caupi com diferentes níveis de severidade da doença, mensurados previamente com o programa AutoCAD $^{\circledR}$, foram avaliados por 10 pessoas, sem e com a utilização da escala diagramática. Foram realizadas duas avaliações com utilização da escala, com intervalo de sete dias, onde seqüências diferentes das mesmas folhas foram estimadas visualmente pelos mesmos avaliadores. A acurácia e a precisão de cada avaliador foram determinadas por regressão linear simples, entre a severidade real, mensurada eletronicamente, e a estimada pelo avaliador. Sem a escala, a maioria dos avaliadores superestimou a severidade da doença. Com a escala, os avaliadores obtiveram melhores níveis de acurácia e precisão, com os erros absolutos concentrando-se na faixa de $10 \%$. Os avaliadores apresentaram boa repetibilidade (90\%) e reprodutibilidade (84\%) das estimativas com a utilização da escala. A escala diagramática proposta demonstrou ser adequada para avaliação da severidade do carvão da folha do caupi.

Palavras-chave adicionais: Vigna unguiculata, Entyloma vignae, doença foliar, patometria. 
O carvão da folha, causado pelo fungo Entyloma vignae Batista et al., é uma importante doença do caupi [Vigna unguiculata (L.) Walp.] nos estados do Nordeste brasileiro, estando presente em todas as áreas onde se cultiva essa leguminosa. Os sintomas da doença se caracterizam pela presença nos folíolos de manchas arredondadas, castanho-escuras, firmes e lisas, podendo atingir até $12 \mathrm{~mm}$ de diâmetro, circundadas por halo clorótico (4). Nos folíolos cloróticos ou amarelecidos, as manchas assumem tonalidade cinza-esverdeada ou olivácea. Em ataques severos, pode haver coalescência das lesões, provocando intenso amarelecimento e queda prematura dos folíolos, com marcante prejuízo ao processo fotossintético e conseqüente redução da produção, sendo estimadas perdas de até $40 \%$ (17).

Embora o carvão da folha do caupi tenha sido reportado mundialmente pela primeira vez no Estado do Ceará há mais de 35 anos (17) e possa induzir severas perdas, até o momento inexistem estudos epidemiológicos dessa doença. Esses estudos requerem métodos padronizados para avaliação da severidade da doença no campo, que devem ser simples e de rápida utilização em condições diferenciadas, bem como acurados, precisos e reprodutíveis (5). A acurácia representa o grau de proximidade entre a estimativa média e a quantidade real de doença, a precisão refere-se à confiabilidade e/ou repetibilidade nas avaliações da doença e a reprodutibilidade indica a ausência de variação em estimativas quando a mesma amostra de doença é avaliada por outro avaliador (6).

$\mathrm{Na}$ avaliação da severidade do carvão da folha do caupi têm sido utilizadas apenas escalas descritivas $(1,2,18,19,20)$, que apresentam a inconveniência de serem altamente subjetivas e não permitirem um ajuste da acuidade visual na avaliação dos níveis de severidade (6). Diante disso, a avaliação da severidade da doença com o auxílio de uma escala diagramática é uma excelente opção para estudos de campo.

$\mathrm{Na}$ elaboração de escalas diagramáticas devem ser considerados importantes aspectos como: o limite superior da escala deve corresponder à intensidade máxima de doença observada no campo; a determinação da intensidade real de doença no campo e sua representação na escala devem ter alta precisão; as subdivisões da escala devem respeitar as limitações da acuidade visual humana definidas pela lei de estímulo-resposta de WeberFechner, na qual a acuidade visual é proporcional ao logaritmo da intensidade do estímulo (10). Além disso, para verificação da qualidade das estimativas propiciadas pela escala diagramática, os níveis de acurácia, precisão e reprodutibilidade devem ser analisados $(6,11,16)$.

Considerando-se a inexistência de métodos padronizados para quantificação do carvão da folha do caupi, este trabalho teve como objetivos desenvolver uma escala diagramática para avaliação da severidade da doença e analisar os níveis de acurácia, precisão e reprodutibilidade das estimativas geradas com sua utilização.

\section{MATERIAL E MÉTODOS}

\section{Elaboração da escala diagramática}

Para elaboração da escala diagramática foram coletados 100 folíolos de caupi (cv. IPA-206) em plantios comerciais no município de Aliança - PE, com diferentes níveis de severidade do carvão da folha. As folhas foram reproduzidas por fotocópias coloridas e digitalizadas. Com auxílio do programa AutoCAD ${ }^{\circledR}$

(Autodesk, 1998), foram determinadas a área foliar total e a área lesionada de cada folha, obtendo-se a severidade da doença (porcentagem de área foliar lesionada). Utilizando o valor máximo de severidade da doença constatada nas folhas coletadas, os intervalos da escala foram calculados com o auxílio do programa 2LOG (22) e os valores obtidos arredondados. Baseando-se na lei de Weber-Fechner de acuidade visual (10), bem como na forma, distribuição e freqüência das lesões, foi confeccionada uma escala diagramática logarítmica com seis níveis de severidade.

\section{Validação da escala diagramática}

No teste de validação da escala diagramática foram utilizados 48 folíolos de caupi com sintomas de carvão em diferentes níveis de intensidade, reproduzidas em fotocópias coloridas. A severidade foi avaliada por 10 pessoas, a maioria com experiência na quantificação de doenças. Inicialmente, a severidade foi estimada sem o auxílio da escala diagramática e, após sete dias, com o auxílio dessa. Visando avaliar a repetibilidade das estimativas com a escala diagramática, sete dias após a primeira avaliação, nova sequiência das mesmas folhas foi organizada e uma segunda estimativa visual com auxílio da escala foi efetuada pelos mesmos avaliadores.

A acurácia e a precisão de cada avaliador foram determinadas por meio de regressão linear simples, com a severidade real obtida eletronicamente como variável independente e a severidade estimada pelo avaliador como variável dependente. A acurácia das estimativas de cada avaliador e do conjunto de avaliadores foi determinada pelo teste $t$ aplicado ao intercepto da regressão linear (a), para verificar a hipótese Ho: $a=0$, e ao coeficiente angular da reta $(b)$, para testar a hipótese Ho: $b=1$, ao nível 5\% de probabilidade $(\mathrm{P}=0,05)$. Valores de intercepto significativamente diferentes de 0 (zero) indicam superestimativa $(>0)$ ou subestimativa $(<0)$ da severidade real a níveis baixos de intensidade da doença, enquanto valores de coeficiente angular da reta que desviam significativamente de 1 (um) indicam superestimativa $(>1)$ ou subestimativa $(<1)$ sistemática da severidade real em todos os níveis de intensidade da doença. A precisão das estimativas foi determinada pelo coeficiente de determinação da regressão $\left(R^{2}\right)$, pela variância dos erros absolutos (severidade estimada menos severidade real) e pela repetibilidade das estimativas, determinada pela regressão da segunda avaliação em relação à primeira de uma mesma unidade amostral. A reprodutibilidade das estimativas foi determinada pelos valores de $R^{2}$ obtidos de regressões lineares entre as severidades estimadas da mesma unidade amostral por diferentes avaliadores combinados em pares $(6,11,16)$. As análises de regressão foram efetuadas com o auxílio do programa Microsoft Excel $2000^{\oplus}$ (Microsoft Corporation, 2000).

\section{RESULTADOS E DISCUSSÃO}

O valor máximo de severidade do carvão nas folhas de caupi coletadas em plantios comerciais foi de 45,0\%. A escala diagramática para quantificação da severidade dessa doença foi elaborada com seis níveis de severidade, representados pelos valores de 1,$5 ; 3,5 ; 7,0 ; 14,5 ; 27,0$ e $45,0 \%$ de área foliar lesionada (Figura 1).

Quando a severidade do carvão da folha do caupi foi estimada sem o auxílio da escala diagramática, $70 \%$ dos avaliadores foram pouco acurados, pois apresentaram valores do intercepto significativamente diferentes de zero para as retas de regressão 


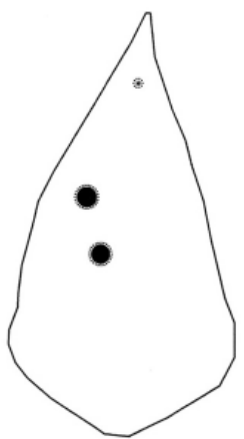

1,5

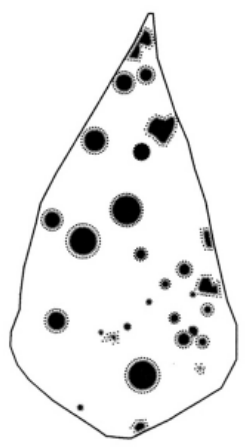

14,5

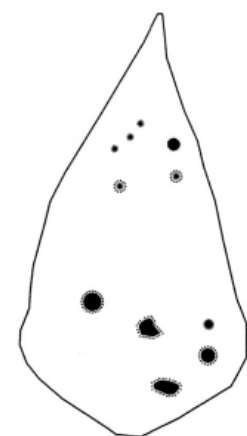

3,5

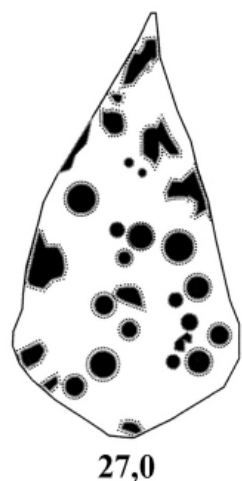

Figura 1. Escala diagramática para avaliação do carvão da folha do

caupi, indicando os níveis de 1,$5 ; 3,5 ; 7,0 ; 14,5 ; 27,0$ e 45,0\% de severidade.
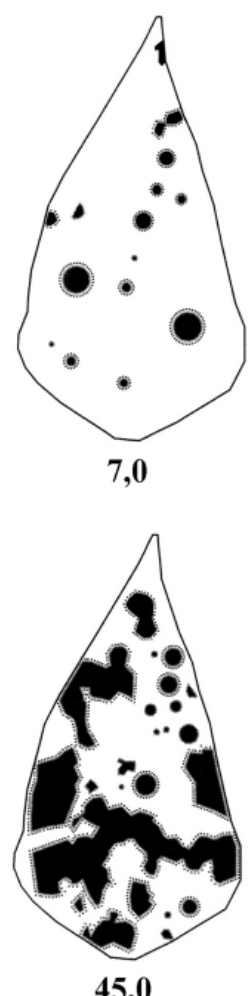

entre severidade real e estimada, com valor médio de 3,82 (Tabela 1). Dentre esses avaliadores, somente um $(\mathrm{H})$ não superestimou consistentemente a severidade, indicando a presença de desvios positivos constantes para a maioria dos avaliadores a baixos níveis de severidade da doença. O coeficiente angular da reta, na média dos avaliadores, foi de 1,04 e não diferiu significativamente de 1 (Tabela 1). No entanto, para 50\% dos avaliadores (A, C, D, G e J) os valores do coeficiente angular foram significativamente diferentes de 1 , indicando a presença de desvios sistemáticos em todo os níveis de intensidade da doença, sendo três avaliadores com tendência à superestimativa $(\mathrm{A}, \mathrm{G}$ e J) e dois à subestimativa (C e D) (Tabela 1). A precisão das estimativas sem o auxílio da escala foi baixa, pois os coeficientes de determinação variaram entre 0,58 e 0,87 , com média de 0,71 . A distribuição dos resíduos das avaliações realizadas sem o auxílio da escala diagramática apresentou estimativas com erros absolutos variando entre $-13,63$ e 38,27 (Figura 2A), sendo a média dos erros de todos os avaliadores igual a 4,22.

Com a utilização da escala diagramática, a maioria dos avaliadores melhorou os níveis de acurácia e precisão das estimativas de severidade do carvão da folha do caupi (Tabela 1). Nas duas avaliações com o auxílio da escala, somente um avaliador (J) apresentou valor do intercepto significativamente diferente de zero. Os valores médios do intercepto na primeira $(0,53)$ e na segunda $(0,70)$ avaliação não diferiram significativamente de zero (Tabela 1), indicando a redução dos erros verificados sem a utilização da escala. Em relação aos valores do coeficiente angular da reta, o uso da escala resultou em coeficientes angulares similares a 1 para todos os avaliadores nas duas avaliações,

Tabela 1. Intercepto $(a)$, coeficiente angular da reta $(b)$ e coeficiente de determinação $\left(R^{2}\right)$ de equações de regressão linear simples relacionando estimativas visuais do carvão da folha do caupi efetuadas por avaliadores, sem e com o auxílio da escala diagramática, à severidade real determinada eletronicamente.

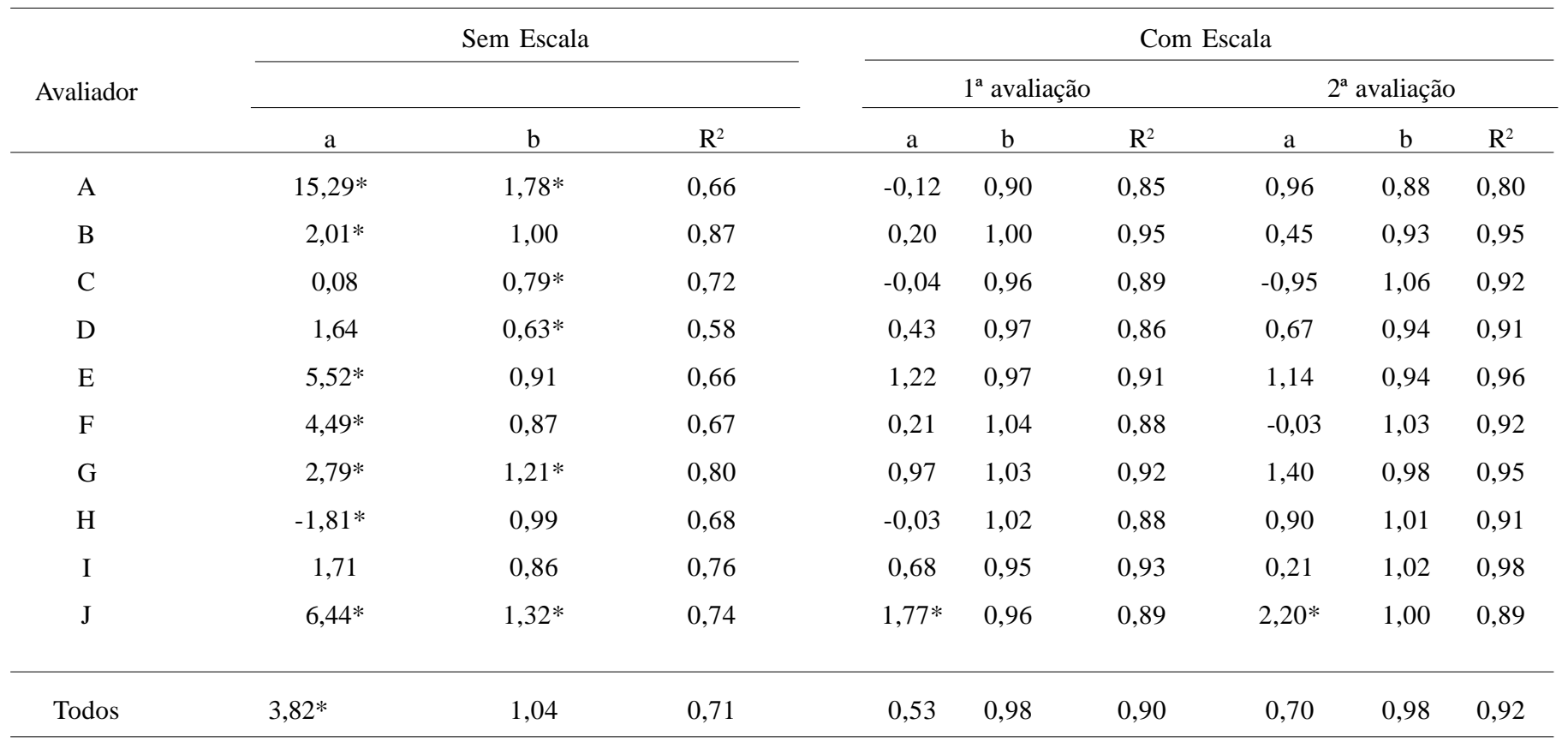

*Asterisco indica que a hipótese de nulidade $(a=0$ ou $b=1)$ foi rejeitada pelo teste $t(\mathrm{P}=0,05)$. 
indicando a redução significativa dos erros sistemáticos das estimativas e melhoria na acurácia das estimativas. Todos os avaliadores melhoraram a precisão das estimativas com a utilização da escala, com coeficientes de determinação variando entre 0,86 e 0,95 na primeira avaliação, e entre 0,80 e 0,98 na segunda, com médias respectivas de 0,90 e 0,92 (Tabela 1), assemelhando-se ao verificados em outros estudos de validação de escalas $(3,7,8$, $9,12,15,22)$. A maioria dos avaliadores melhorou a precisão das estimativas na segunda avaliação com a escala em relação à primeira, indicando que além da utilização da escala diagramática, a familiarização dos avaliadores com a doença e o treinamento destes pode melhorar a acurácia e a precisão das estimativas (14, 22).

Houve redução nos erros absolutos para as estimativas com o auxílio da escala diagramática (Figuras $2 \mathrm{~B}$ e 2C), quando comparada com a distribuição dos resíduos das estimativas obtidas sem a escala (Figura 2A). Com a escala, os erros absolutos não

Tabela 2. Intercepto $(a)$, coeficiente angular da reta $(b)$ e coeficiente de determinação $\left(R^{2}\right)$ de equações de regressão linear simples relacionando a segunda à primeira estimativa do carvão da folha do caupi por um mesmo avaliador, com o auxílio da escala diagramática.

\begin{tabular}{cccc}
\hline Avaliador & $a$ & $b$ & $R^{2}$ \\
\hline A & 1,83 & 0,90 & 0,79 \\
B & 0,63 & 0,90 & 0,93 \\
C & $2,02^{*}$ & $0,81^{*}$ & 0,88 \\
D & 1,07 & 0,89 & 0,90 \\
E & 0,78 & 0,90 & 0,92 \\
F & 0,23 & 0,95 & 0,96 \\
G & 1,34 & $0,88^{*}$ & 0,89 \\
H & 1,54 & 0,93 & 0,92 \\
I & 0,05 & 1,02 & 0,95 \\
J & 1,11 & 0,98 & 0,89 \\
\hline Média & 1,06 & 0,92 & 0,90 \\
\hline
\end{tabular}

*Asterisco indica que a hipótese de nulidade $(a=0$ ou $b=1)$ foi rejeitada pelo teste $t(\mathrm{P}=0,05)$. apresentaram um padrão indesejável definido e variaram na primeira avaliação entre -10,63 e 10,68 (Figura 2B), com média de 0,31 , enquanto na segunda avaliação entre -7,63 e 15,15 (Figura $2 \mathrm{C}$ ), com média de 0,45 . A maioria dos erros absolutos dos avaliadores que utilizaram a escala foi inferior a $10 \%$, considerado bom segundo os critérios adotados em vários estudos de avaliação de escalas diagramáticas $(3,7,8,9,12,13,14,15,22)$. A presença de algum nível de erro absoluto nas mensurações pode ser compensada pela rapidez e padronização propiciadas pelo uso de escalas diagramáticas. Além disso, como a maioria dos métodos de quantificação de severidade de doenças, o uso de escalas diagramáticas está sujeito a um certo grau de subjetividade, o que pode ser minimizado com o treinamento dos avaliadores (16).

A diferença entre os avaliadores na mensuração do carvão da folha do caupi confirma as observações de Nutter \& Schultz (16) quanto à variação na habilidade entre indivíduos para discriminar níveis de doença. A qualidade da estimativa da doença, além de ser influenciada por estímulos e respostas psicológicas, pode ser afetada por fatores como complexidade da unidade amostral, tamanho e forma das lesões, cor e número de lesões na unidade amostral (11), fadiga, dificuldade de concentração na tarefa (21) e experiência de cada indivíduo na avaliação da doença $(5,14$, 22).

Em geral, os avaliadores evidenciaram boa repetibilidade nas estimativas da severidade do carvão da folha do caupi com o uso da escala diagramática, pois a quantidade média de variação na primeira avaliação explicada pela segunda avaliação foi de $90 \%$ (Tabela 2). Os valores médios do intercepto e do coeficiente angular da reta não diferiram significativamente de zero e 1 , respectivamente. Entre as duas avaliações, somente para um avaliador $(\mathrm{C}) \mathrm{o}$ valor do intercepto foi significativamente diferente de zero e para dois avaliadores $(\mathrm{C} \mathrm{e} \mathrm{G})$ os valores do coeficiente angular da reta foram significativamente diferentes de 1 , indicando a elevada precisão das estimativas para a maioria dos avaliadores.

A reprodutibilidade das avaliações da severidade sem a utilização da escala diagramática foi baixa, pois as regressões lineares entre as severidades estimadas pelos 10 avaliadores produziram coeficientes de determinação variando de 0,30 e 0,84 , sendo que em somente $15,6 \%$ dos casos foi $\geq 0,75$ (Tabela 3 ).
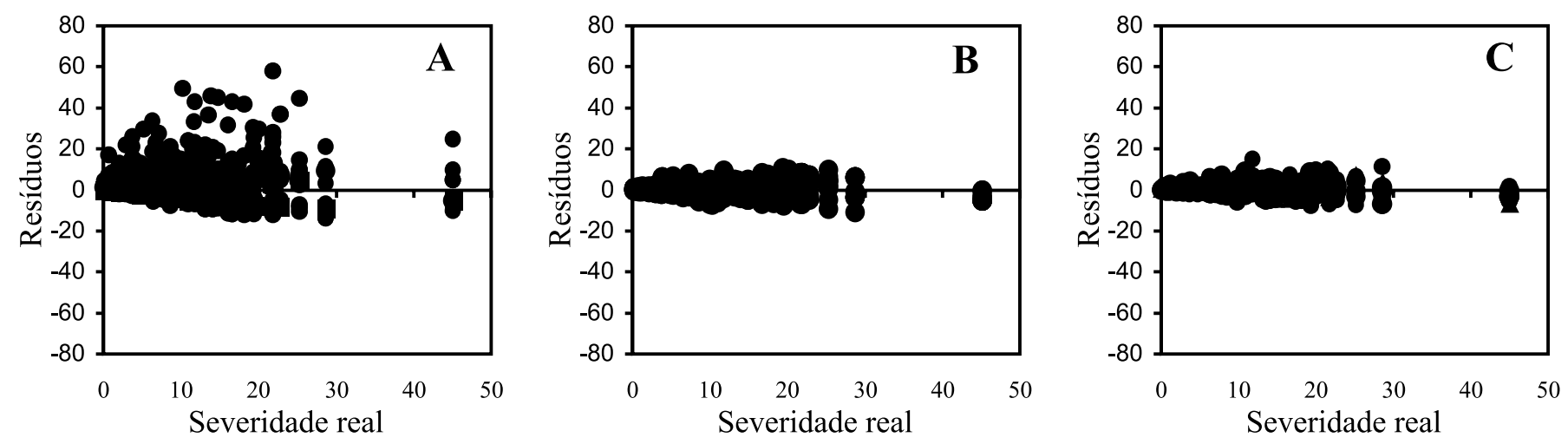

Figura 2. Resíduos (severidade estimada - severidade real) das estimativas do carvão da folha do caupi, realizadas sem o auxílio da escala diagramática (A) e com auxílio da escala diagramática, na primeira (B) e segunda (C) avaliação. 
Por outro lado, com a utilização da escala diagramática as avaliações apresentaram elevada reprodutibilidade, pois nas duas avaliações os valores do coeficiente de determinação foram elevados, variando entre 0,68 a 0,95 , sendo $\geq 0,75 \mathrm{em} 88,9 \%$ dos casos na primeira avaliação e em 93,3\% dos casos na segunda (Tabela 3), assemelhado-se ao constado na validação de escalas diagramáticas para outros patossistemas $(7,8,12,13,22)$. O uso da escala diagramática aumentou a reprodutibilidade das

Tabela 3. Coeficiente de determinação $\left(R^{2}\right)$ de equações de regressão linear simples relacionando as estimativas do carvão da folha do caupi entre avaliadores sem o auxílio da escala diagramática e com auxílio da escala diagramática na primeira e segunda avaliação.

\begin{tabular}{|c|c|c|c|c|c|c|c|c|c|}
\hline \multicolumn{10}{|c|}{ Sem Escala } \\
\hline Avaliador & B & $\mathrm{C}$ & D & $\mathrm{E}$ & $\mathrm{F}$ & G & $\mathrm{H}$ & I & $\mathrm{J}$ \\
\hline A & 0,68 & 0,30 & 0,52 & 0,67 & 0,73 & 0,72 & 0,39 & 0,65 & 0,80 \\
\hline B & & 0,62 & 0,63 & 0,71 & 0,71 & 0,83 & 0,71 & 0,84 & 0,75 \\
\hline C & & & 0,44 & 0,33 & 0,35 & 0,41 & 0,72 & 0,60 & 0,43 \\
\hline D & & & & 0,65 & 0,53 & 0,57 & 0,48 & 0,65 & 0,51 \\
\hline E & & & & & 0,64 & 0,73 & 0,52 & 0,70 & 0,62 \\
\hline $\mathrm{F}$ & & & & & & 0,63 & 0,42 & 0,56 & 0,76 \\
\hline G & & & & & & & 0,56 & 0,76 & 0,69 \\
\hline $\mathrm{H}$ & & & & & & & & 0,77 & 0,49 \\
\hline I & & & & & & & & & 0,64 \\
\hline
\end{tabular}

Com Escala - $1^{\mathrm{a}}$ Avaliação

\begin{tabular}{ccccccccccc}
\hline Avaliador & B & C & D & E & F & G & H & I & J \\
A & 0,89 & 0,71 & 0,74 & 0,81 & 0,79 & 0,80 & 0,74 & 0,84 & 0,77 \\
B & & 0,84 & 0,87 & 0,92 & 0,90 & 0,91 & 0,83 & 0,92 & 0,89 \\
C & & & 0,73 & 0,85 & 0,76 & 0,78 & 0,77 & 0,84 & 0,80 \\
D & & & & 0,81 & 0,85 & 0,90 & 0,73 & 0,80 & 0,81 \\
E & & & & & 0,83 & 0,87 & 0,80 & 0,89 & 0,81 \\
F & & & & & 0,85 & 0,85 & 0,88 & 0,85 \\
G & & & & & & 0,82 & 0,87 & 0,85 \\
H & & & & & & & & & 0,82 & 0,78 \\
I & & & & & & & & & & 0,87 \\
\hline
\end{tabular}

Com Escala - 2a Avaliação

\begin{tabular}{ccccccccccc}
\hline Avaliador & B & C & D & E & F & G & H & I & J \\
A & 0,80 & 0,70 & 0,71 & 0,80 & 0,80 & 0,78 & 0,68 & 0,79 & 0,72 \\
B & & 0,88 & 0,89 & 0,95 & 0,91 & 0,89 & 0,86 & 0,90 & 0,85 \\
C & & & 0,82 & 0,89 & 0,82 & 0,85 & 0,8, & 0,90 & 0,78 \\
D & & & & 0,88 & 0,84 & 0,88 & 0,85 & 0,88 & 0,81 \\
E & & & & 0,88 & 0,91 & 0,87 & 0,93 & 0,85 \\
F & & & & & 0,89 & 0,90 & 0,89 & 0,89 \\
G & & & & & & 0,88 & 0,95 & 0,87 \\
H & & & & & & & & & 0,91 & 0,83 \\
I & & & & & & & & & & 0,91 \\
\hline
\end{tabular}


estimativas obtidas por diferentes avaliadores e, do ponto de vista prático, diferentes experimentos, conduzidos por avaliadores distintos, serão comparáveis desde que utilizem a escala diagramática.

Os níveis de acurácia, precisão e reprodutibilidade das mensurações da severidade do carvão da folha do caupi melhoraram significativamente com o uso da escala diagramática, indicando que em estudos epidemiológicos poderá proporcionar informações mais realistas a respeito do patossistema Entyloma vignae - caupi e melhorar as avaliações das estratégias de controle do carvão da folha.

\section{AGRADECIMENTOS}

Os autores expressam seus agradecimentos a todos os avaliadores que participaram das etapas de validação da escala diagramática.

\section{REFERÊNCIAS BIBLIOGRÁFICAS}

1. Adejumo, T.O.; Florini, D.A.; Ikotun, T. Screening of cowpea cultivars for resistance to leaf smut. Crop Protection, Oxford, v.20, n.4, p.303-309, 2001.

2. Almeida, R.T.; Vasconcelos, I.; Paiva, J.B.; Souza, C.A.V.; Freire, V.F. Avaliação da incidência de Entyloma vignae sobre cultivares e progênies de feijão-de-corda, Vigna sinensis. Ciência Agronômica, Fortaleza, v.10, n.1, p.31-34, 1970.

3. Amorim, L.; Bergamin Filho, A.; Palazzo, D.A.; Bassanezi, R.B.; Godoy, C.V.; Torres, G.M. Clorose variegada dos citros: uma escala diagramática para avaliação da severidade da doença. Fitopatologia Brasileira, Brasília, v.18, n.2, p.174-180, 1993.

4. Athayde Sobrinho, C.; Viana, F.M.P.; Santos, A.A. doenças do feijão caupi. In: Cardoso, M.J. (Org.) A cultura do feijão caupi no Meio-Norte do Brasil. Teresina: Embrapa Meio-Norte, 2000. p.157-183. (Circular Técnica, 28).

5. Berger, R.D. Measuring disease intensity. In: Teng, P.S.; Krupa, S.V. (Ed.) Crop loss assessment. St. Paul: University Minnesota, 1980. p.28-31.

6. Campbell, C.L.; Madden, L.V. Introduction to plant disease epidemiology. New York: John Wiley, 1990. 532p.

7. Díaz, C.G.; Bassanezi, R.B.; Bergamim Filho, A. Desenvolvimento e validação de uma escala diagramática para Xanthomonas axonopodis pv. phaseoli em feijoeiro. Summa Phytopathologica, Jaboticabal, v.27, n.1, p.35-39, 2001.

8. Godoy, C.V.; Carneiro, S.M.T.P.G.; Iamuti, M.T.; Dalla Pria, M. Amorim, L.; Berger, R.D.; Bergamim Filho, A. Diagramatic scale for bean: development and validation. Zeitschrift für Pflanzenkrankheiten und Pflanzenschutz, Stuttgart, v.104, n.4, p.336-345, 1997.

9. Gomes, A.M.A.; Michereff, S.J.; Mariano, R.L.R. Elaboração e validação de escala diagramática para cercosporiose da alface. Sum- ma Phytopathologica, Botucatu, v.30, n.1, p.39-43, 2004.

10. Horsfall, J.G.; Cowling, E.B. Pathometry: the measurement of plant disease. In: Horsfall, J.G.; Cowling, E.B. (Ed.) Plant disease: an advanced treatise - how disease develops in populations. New York: Academic Press, 1978. v.2, p.119-136.

11. Kranz, J. Measuring plant disease. In: Kranz, J.; Rotem, J. (Ed.) Experimental techniques in plant disease epidemiology. Heidelberg: Springer-Verlag, 1988. p.35-50.

12. Leite, R.M.V.B.C.; Amorim, L. Elaboração e validação de escala diagramática para mancha de Alternaria em girassol. Summa Phytopathologica, Botucatu, v. 28, n.1, p.14-19, 2002.

13. Martins, M.C.; Guerzoni, R.A.; Câmara, G.M.S.; Mattiazzi, P.; Lourenço, S.A.; Amorim, L. Escala diagramática para a quantificação do complexo de doenças foliares de final de ciclo em soja. Fitopatologia Brasileira, Fortaleza, v.29, n.2, p.179-184, 2004.

14. Michereff, S.J.; Maffia, L.A.; Noronha, M.A. Escala diagramática para avaliação da severidade da queima das folhas do inhame. Fitopatologia Brasileira, Brasília, v. 25, n.4, 612-619, 2000.

15. Michereff, S.J.; Pedrosa, R.A.; Noronha, M.A.; Martins, R.B.; Silva, F.V. Escala diagramática e tamanho de amostras para avaliação da severidade da mancha parda da mandioca (Cercosporidium henningsii). Agrotrópica, Itabuna, n.3, p.143-148, 1998.

16. Nutter Jr., F.W.; Schultz, P.M. Improving the accuracy and precision of disease assessments: selection of methods and use of computer-aided training programs. Canadian Journal of Plant Pathology, Ottawa, v.17, n.1, p.174-184, 1995.

17. Ponte, J.J. Uma nova doença do feijão-de-corda, Vigna sinensis Endl. Boletim da Sociedade Cearense de Agronomia, Fortaleza, v.7, n.1, p.35-38, 1966.

18. Ponte, J.J.; Vasconcelos, I.; Paiva, J.B.; Castro, F.E.; Sobral, C.A.M. Incidência do carvão (Entyloma vignae) em cultivares de feijão macassar (Vigna sinensis), procedentes da Nigéria. Summa Phytopathologica, Piracicaba, v.2, n.1, p.50-52, 1976.

19. Prabhu, A.S.; Albuquerque, F.C.; Lima, E.F. Avaliação da resistência a Entyloma vignae em feijão caupi. Fitopatologia Brasileira, Brasília, v.4, n.3, p.375-378, 1979.

20. Santos, A.A.; Quinderé, M.A.W.; Melo, M.B. Avaliação de genótipos de caupi para resistência ao carvão (Entyloma vignae). Fitopatologia Brasileira, Brasília, v.22, n.1, p.77-78, 1997.

21. Shokes, F.M.; Berger, R.D.; Smith, D.H.; Rasp, J.M. Reliability of disease assessment procedures: a case study with late leafspot of peanut. Oléagineux, Paris, v.42, n.4, p.245-251, 1987.

22. Tovar-Soto, A.; Hernandez-Martínez, M.; Cristóbal-Alejo, J.; Romero-Hijo, R.; Mora-Aguilera, G. Escala logarítimica diagramática de severidad de la mancha negra (Colletotrichum gloeosporioides Penz.) en Chirimoyo (Annona cherimola Mill). Revista Mexicana de Fitopatología, Sonora, v.20, n.1, p.103-109, 2002. 